\title{
Anal cancer and intraepithelial neoplasia: epidemiology, screening and prevention of a sexually transmitted disease
}

\author{
Richard J. Medford MD, Irving E. Salit MD
}

See also CMAJ Open research article at www.cmajopen.ca/content/2/3/E127.full

$\mathrm{A}$ nal cancer is generally uncommon, ${ }^{1}$ but some groups at high risk have higher prevalence. It is usually caused by the human papillomavirus (HPV) and can occur along with other HPV-related cancers in the same patient or in his or her sexual partners. Anal cancer is potentially preventable, and precancers can be detected and treated in the office.

There were 580 cases of anal cancer in Canada in 2010, 195 (33.6\%) of which occurred in men. ${ }^{1}$ Although anal cancer is more common in women, ${ }^{1,2}$ high rates of the disease are developing among people with HIV infection, particularly among men who have sex with men. ${ }^{3,4}$

Similar to cervical cancer, $80 \%-90 \%$ of anal cancers are caused by HPV, and premalignant changes, termed anal intraepithelial neoplasias (AINs), occur. ${ }^{5,6}$ Although screening for anal cancer and its precursors is recommended, ${ }^{7,8}$ there are no consensus guidelines for doing so in Canada.

The incidence of anal cancer in Canada is 1.7 per 100000 person-years. ${ }^{1,9}$ However, among people with HIV infection, the rates range from 49 to 144 per 100000 person-years. ${ }^{3,4,10}$ This figure is comparable to the rate of colorectal cancer in men (60 per 100000 person-years). ${ }^{1}$ Among 13 cohorts, unadjusted rates of anal cancer were 131 , 46 and 2 per 100000 for men with HIV infection who have sex with men (rate ratio [RR] 80.3, 95\% confidence interval [CI] 42.7-151.1), men with HIV infection (RR 26.7, 95\% CI 11.5-61.7) and men without HIV infection, respectively. ${ }^{11}$

Here, we review the epidemiology, screening and prevention of anal cancer. We conducted a broad literature search to inform this review, and the level of evidence ranged from expert opinion to randomized controlled trials (Box 1). The term anal cancer as used in this review refers to the predominant histologic form of anal cancer, which is squamous cell carcinoma.

\section{How are anal cancers classified, and what are the risk factors?}

The anal canal extends $4 \mathrm{~cm}$ from the anorectal junction caudally to the anal verge, beyond which is the anal margin or perianal skin. The canal is lined by squamous tissue that transitions to the columnar or glandular tissue of the rectum at the squamo-columnar junction. Squamous cell carcinoma and precancerous lesions (i.e., AINs) are most likely to arise at this junction. Some squamous cancers also occur in the perianal area, but they behave more like skin cancers. ${ }^{12}$ In this review, we will focus on squamous cell carcinoma of the anal canal, for which risk factors (Box 2) ${ }^{12}$ overlap with those for HPV infection.

Oncogenic strains of HPV, particularly types 16 and 18 , cause $80 \%-90 \%$ of anal cancers. HPV encodes tumour suppressor proteins E6 and E7, and their interactions with intracellular proteins p53 and retinoblastoma lead to changes in cell growth and regulation, apoptosis and immortalization leading to malignant disease. ${ }^{13,14}$

\section{What is the connection between immune suppression and anal cancer?}

With the advent of combined antiretroviral treatment, people with HIV infection are living longer, and AIDS-defining cancers such as Kaposi sarcoma, non-Hodgkin lymphoma and cervical cancer have declined. However, non-AIDS defining cancers are increasing in this population. ${ }^{10,15-17}$ Despite very effective antiretroviral treatments, there has been a progressive increase in the incidence of anal cancer, which appears to have plateaued recently. $3,4,11,16,18-21$

\section{KEY POINTS}

- Anal cancer and anal intraepithelial neoplasia are sexually transmitted diseases associated with certain oncogenic strains of human papillomavirus (HPV), particularly strains 16 and 18 .

- Anal cancer is prevalent among people with HIV infection, particularly men who have sex with men.

- Populations at high risk of acquiring anal cancer should undergo regular screening, although the efficacy of this approach has not yet been proven in clinical trials.

- The HPV vaccine is effective in reducing rates of anal intraepithelial neoplasia and anal cancer. 
In one large study, there was no relationship between CD4+ T helper cell counts at HIV registration and risk of anal cancer. ${ }^{22}$ Conversely, other studies have reported associations between lower CD4+ $\mathrm{T}$ helper cell counts at baseline or nadir CD4+ T helper cell counts and anal cancer. ${ }^{21,23}$ Mortality from non-AIDS defining cancers decreased from 6.0 (95\% CI 3.3-10.1) to 0.6 (95\% CI $0.4-0.8)$ per 1000 person-years when the CD4+ T helper cell count rose to more than 500 cells $/ \mu \mathrm{L} .{ }^{24}$ Prolonged undetectable HIV viral loads were also associated with a decreased risk of anal cancer. ${ }^{25}$

Recipients of solid organ transplantation also have an increased incidence of HPV-related cancers, including anal cancer (11.6 per 100000 person-years, increased standardized incidence ratio $2.77-10.26) .{ }^{26,27}$

\section{Should we screen for premalignant lesions, and if so, how?}

Lack of evidence that screening reduces rates of anal cancer and mortality has led to a lack of guidelines for screening. Uncertainty exists as to which at-risk populations should undergo screening, and there are differing views on the cost-effectiveness of screening and variable screening methods.

The idea behind screening for anal cancer is to detect precancerous histopathologic changes (dysplasia) and ablate these lesions before invasive cancer occurs, as with screening for cervical cancer.

Screening can involve an initial anal Papanicolaou (Pap) test or cytology using a swab inserted into the anal canal past the squamocolumnar junction. The swab is vigorously rotated to acquire cells, then placed in liquidbased medium for cytologic examination. The same sample can be used to test for HPV. A digital anorectal exam is then performed to detect

\section{Box 1: Evidence used for this review}

We performed a PubMed and Embase literature search from the earliest possible date to June 2014. We used the search terms "anal cancer" and "anal intraepithelial neoplasia" with the following medical subject headings to identify the most relevant research: "HIV," "incidence," "HPV," "HPV vaccine," "treatment," "HAART," "CART," "trends," "cigarette smoking," and "solid organ transplantation." We manually reviewed the abstracts and bibliographies of relevant studies to identify additional articles. We included all types of reports (randomized controlled trials, meta-analyses, reviews and prospective cohort, retrospective cohort, casecontrol and cross-sectional studies). We included only articles in English. Where possible, we selected the most recent articles (published within the last $5 \mathrm{yr}$ ) with the highest level of evidence (e.g., randomized controlled trials; meta-analysis of randomized controlled trials) for inclusion. We reviewed 232 articles for relevance, 49 of which are included in this review. warts, induration or masses. If the results of the Pap test are abnormal, high-resolution anoscopy is typically done to examine the anal canal, with a bright light and magnification being provided by a colposcope. Staining with vinegar and Lugol's iodine aids identification of possible dysplastic lesions for biopsy. The pathology grading system mimics that of cervical pathology. For cytology, this includes atypical squamous cells of undetermined significance (ASCUS), low-grade squamous intraepithelial lesions (LSILs) and high-grade squamous intraepithelial lesions (HSILs). For histology, the lesions can be designated as either low-grade AIN (AIN 1; LSIL) or high-grade AIN (AIN 2/3; HSIL).

Although some clinicians do anal cytology first and proceed to high-resolution anoscopy only if the cytology is abnormal, others go directly to high-resolution anoscopy because cytology has low specificity, and high-resolution anoscopy with directed biopsy is the gold standard for detecting AIN lesions (Figure 1). ${ }^{28,29}$ The preferred approach depends on the population undergoing screening and the resources and expertise available.

The New York State Department of Health AIDS Institute and European guidelines suggest annual anal Pap screening of men with HIV infection who have sex with men, with referral for high-resolution anoscopy for any abnormal cytology. ${ }^{7,829}$ The reason for this approach is the high sensitivity of Pap screening at this cytologic cutoff $(84 \%)$. However, the specificity is only $39 \%,{ }^{31}$ with a positive likelihood ratio of 1.38 and negative likelihood ratio of 0.41 .

If a small number of people at high risk are undergoing screening and the resources are available, some experts prefer to go directly to highresolution anoscopy without cytology. ${ }^{29}$ However, the lack of experienced providers to perform the procedures and the associated costs do not allow for the screening of large at-risk populations using this approach.

Box 2: Risk factors for anal cancer ${ }^{12}$

- Human papillomavirus infection

- HIV infection*

- Multiple sexual partners*

- Anal-receptive intercourse*

- Anal warts

- History of cervical, vulvar or vaginal carcinoma

- Chronic immune suppression not caused by HIV infection*

- Cigarette smoking

*Risk factors that overlap with those for HPV infection. 
If many patients are being offered screening, triaging by cytology is likely the best approach. Anoscopy should then be reserved for those with HSIL on cytology because the specificity approaches $91 \% .^{31}$

Whether cytology or high-resolution anoscopy is used initially, a threshold of any abnormal cytology leads to unnecessary high-resolution anoscopies, whereas using an HSIL threshold misses patients with internal highgrade disease. The addition of biomarkers such as HPV typing or E6 RNA transcripts may help to better determine who should undergo highresolution anoscopy. ${ }^{32}$

Digital anal examination should be emphasized as a method for detecting early cancers, because these smaller cancers have a better outcome. However, a digital examination does not detect precancerous lesions.
There have been no clinical trials showing the utility of screening for anal cancer precursors. Although much of the evidence is drawn through parallels to screening for cervical cancer, progression from anal dysplasia to cancer may not occur at the same rate as the progression to cervical cancer. ${ }^{33}$ About $30 \%$ of inadequately treated cervical intraepithelial neoplasia progresses to cancer over 30 years. ${ }^{34}$ In comparison, studies have shown rates of progression of highgrade dysplasia to anal cancer in men with HIV who have sex with men to be around $15 \%$ over two to five years. ${ }^{35,36}$ Others, however, have calculated the current progression rate to be slower $(0.3 \%$ per year $){ }^{30}$

An Ontario study looking at the costeffectiveness of detecting AIN 2/3 in men with HIV who have sex with men concluded that direct use of high-resolution anoscopy without

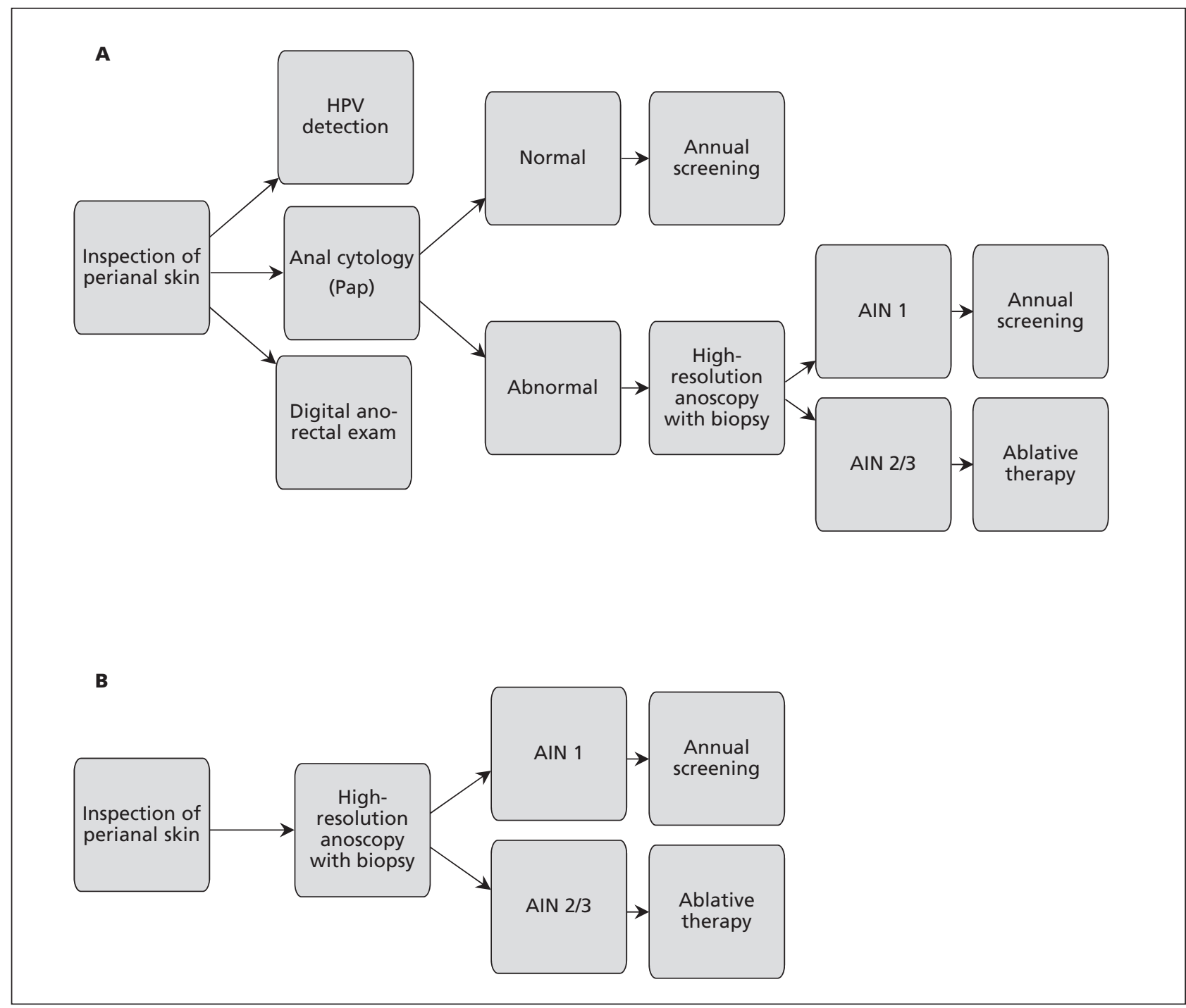

Figure 1: Two approaches to screening for anal cancer. (A) High-resolution anoscopy with abnormal results on anal cytology. (B) High-resolution anoscopy done directly. Adapted from Lam et al. ${ }^{29}$ and Palefsky and Rubin. ${ }^{30}$ Note: AIN = anal intraepithelial neoplasia, HPV = human papillomavirus, Pap = Papanicolaou test. 
preceding cytology was the best strategy. Although this approach was initially more costly, the expense was offset by a higher sensitivity and the low specificity of both HPV and cytology testing. ${ }^{29}$ A recent study confirmed these findings, with AIN 2/3 diagnosed in 28 patients $(52.7 \%$ ) who did not have HSIL on anal cytology. ${ }^{37}$ The approvpriate screening interval is unclear: an American systematic review showed that screening for anal cancer among men with HIV who have sex with men every one to two years was cost-effective; this result was not confirmed in the United Kingdom. ${ }^{38}$

Screening for anal cancer in populations at high risk is acceptable and feasible. ${ }^{39}$ In addition to men with HIV who have sex with men, we suggest that other groups at risk should also undergo screening, including men and women with HIV, women with genital dysplasia or cancers, people with perianal high-grade lesions, and recipients of solid organ transplants.

\section{Is anal cancer preventable?}

Prevention can be categorized according to behavioural and nonbehavioural interventions (Box 3). Safer sex practices, such as a reduced number of partners and regular condom use, can reduce the risk of HPV-related diseases ${ }^{40}$ and facilitates clearance of HPV-associated penile lesions in men whose partners have cervical dysplasia ${ }^{41}$ Cigarette smoking is linked to anal cancer and cytologic abnormalities. ${ }^{42,43}$ There is some protection against AIN in patients with HIV who have high CD4+ T helper cell counts and controlled viral loads. ${ }^{25}$ Male circumcision reduces penile oncogenic HPV infection, markedly reduces the risk of penile cancer and reduces the risk of cervical cancer in female partners. ${ }^{44,45}$ High consumption of fruits, vegetables, vitamins and minerals with antioxidant properties helps to reduce progression of HPV infections to high-grade lesions. ${ }^{46}$

The quadrivalent HPV vaccine (types 6, 11, 16 and 18) protects against high-grade cervical lesions $^{47,48}$ and against high-grade anal dysplasia

\footnotetext{
Box 3: Preventing anal cancer

Behavioural interventions

- Safer sex practices (i.e., condom use) $)^{40}$

- Smoking cessation ${ }^{42,43}$

- Diet modification ${ }^{46}$

Nonbehavioural interventions

- Male circumcision ${ }^{44,45}$

- Vaccination against human papillomavirus ${ }^{47,48}$
}

among men who have sex with men..$^{49}$ Rates of high-grade dysplasia related to the types of HPV against which the vaccine protects were reduced by $54.2 \%$ (95\% CI 18.0-75.3) in the intention-totreat population and by $74.9 \%$ (95\% CI 8.8-95.4) in the per-protocol population. ${ }^{49}$ The vaccine also reduced persistent anal infection. In addition to its role in preventing cervical cancer among women, the quadrivalent HPV vaccine is recommended in Canada for boys and men between the ages of 9 and 26 years for the prevention of AIN grades 1-3, anal cancer and anogenital warts. ${ }^{9}$ The bivalent vaccine (types 16 and 18) has been proven to help protect against high-grade cervical lesions; however, there have been no randomized controlled trials looking at the bivalent vaccine in preventing high-grade anal dysplasia.

It seems intuitive that, in populations with high rates of anal cancer, high-grade lesions are preinvasive and should be removed. In clinics where screening and ablation are done, anal cancers are rarely seen. ${ }^{50,51}$

\section{Gaps in knowledge}

Although screening and early treatment should help prevent anal cancer, similar to what has been accomplished with screening for cervical cancer, the efficacy of this approach has not yet been proven in clinical trials.

\section{Conclusion}

Anal cancer is a preventable sexually transmitted disease. It is important to recognize populations at risk and counsel patients on early detection, safer sex practices, smoking cessation and HPV vaccination. The current quadrivalent HPV vaccine prevents AIN, anal cancer and anogenital warts.

\section{References}

1. Anal cancer statistics. Toronto: Canadian Cancer Society; 2013. Available: www.cancer.ca/en/cancer-information/cancer-type /anal/statistics/?region=on (accessed 2014 July 20).

2. Siegel R, Naishadham D, Jemal A. Cancer statistics, 2013. $C A$ Cancer J Clin 2013;63:11-30.

3. Crum-Cianflone NF, Hullsiek KH, Marconi VC, et al. Anal cancers among HIV-infected persons: HAART is not slowing rising incidence. AIDS 2010;24:535-43.

4. D'Souza G, Wiley DJ, Li X, et al. Incidence and epidemiology of anal cancer in the multicenter AIDS cohort study. $J$ Acquir Immun Defic Syndr 2008;48:491-9.

5. De Vuyst H, Clifford GM, Nascimento MC, et al. Prevalence and type distribution of human papillomavirus in carcinoma and intraepithelial neoplasia of the vulva, vagina and anus: a meta-analysis. Int J Cancer 2009;124:1626-36.

6. Parkin DM. The global health burden of infection-associated cancers in the year 2002. Int J Cancer 2006;118:3030-44.

7. HIV clinical resource: anal dysplasia and cancer. New York: Office of the Medical Director New York State Department of Health AIDS Istitute; 2007.

8. EACS guidelines: version 7.02. Brussels (Belgium): European AIDS Clinical Society; 2014.

9. National Advisory Committee on Immunization. Update on human papillomavirus (HPV) vaccines. An Advisory Committee statement. Can Comm Dis Rep 2012;38:ACS-1. Available: www.phac 
-aspc.gc.ca/publicat/ccdr-rmtc/12vol38/acs-dcc-1/index-eng.php\# a3-2 (accessed 2014 Nov. 19).

10. Bedimo R, Chen RY, Accortt NA, et al. Trends in AIDS-defining and non-AIDS-defining malignancies among HIV-infected patients: 1989-2002. Clin Infect Dis 2004;39:1380-4.

11. Silverberg MJ, Lau B, Justice AC, et al. Risk of anal cancer in HIV-infected and HIV-uninfected individuals in North America. Clin Infect Dis 2012;54:1026-34.

12. Welton ML, Sharkey FE, Kahlenberg MS. The etiology and epidemiology of anal cancer. Surg Oncol Clin N Am 2004;13: 263-75.

13. Stelzer MK, Pitot HC, Liem A, et al. A mouse model for human anal cancer. Cancer Prev Res (Phila) 2010;3:1534-41.

14. Thomas MK, Pitot HC, Liem A, et al. Dominant role of HPV16 E7 in anal carcinogenesis. Virology 2011;421:114-8.

15. Clifford GM, Polesel J, Rickenbach M, et al. Cancer risk in the Swiss HIV Cohort Study: associations with immunodeficiency, smoking, and highly active antiretroviral therapy. J Natl Cancer Inst 2005;97:425-32.

16. Diamond C, Taylor TH, Aboumrad T, et al. Increased incidence of squamous cell anal cancer among men with AIDS in the era of highly active antiretroviral therapy. Sex Transm Dis 2005;32:314-20.

17. Engels EA, Pfeiffer RM, Goedert JJ, et al. Trends in cancer risk among people with AIDS in the United States 1980-2002. AIDS 2006;20:1645-54

18. Piketty C, Selinger-Leneman H, Grabar S, et al. Marked increase in the incidence of invasive anal cancer among HIVinfected patients despite treatment with combination antiretroviral therapy. AIDS 2008;22:1203-11.

19. Hessol NA, Pipkin S, Schwarcz S, et al. The impact of highly active antiretroviral therapy on non-AIDS-defining cancers among adults with AIDS. Am J Epidemiol 2007;165:1143-53.

20. Simard EP, Pfeiffer RM, Engels EA. Cumulative incidence of cancer among individuals with acquired immunodeficiency syndrome in the United States. Cancer 2011;117:1089-96.

21. Patel P, Hanson DL, Sullivan PS, et al. Incidence of types of cancer among HIV-infected persons compared with the general population in the United States, 1992-2003. Ann Intern Med 2008;148:728-36.

22. Engels EA, Biggar RJ, Hall HI, et al. Cancer risk in people infected with human immunodeficiency virus in the United States. Int J Cancer 2008;123:187-94.

23. Bedimo RJ, McGinnis KA, Dunlap M, et al. Incidence of nonAIDS-defining malignancies in HIV-infected versus noninfected patients in the HAART era: impact of immunosuppression. J Acquir Immune Defic Syndr 2009;52:203-8.

24. Monforte A, Abrams D, Pradier C, et al. HIV-induced immunodeficiency and mortality from AIDS-defining and nonAIDS-defining malignancies. AIDS 2008;22:2143-53.

25. Chiao EY, Hartman CM, El-Serag HB, et al. The impact of HIV viral control on the incidence of HIV-associated anal cancer. J Acquir Immune Defic Syndr 2013;63:631-8.

26. Engels EA, Pfeiffer RM, Fraumeni JF Jr, et al. Spectrum of cancer risk among US solid organ transplant recipients. JAMA 2011;306:1891-901.

27. Grulich AE, van Leeuwen MT, Falster MO, et al. Incidence of cancers in people with HIV/AIDS compared with immunosuppressed transplant recipients: a meta-analysis. Lancet 2007; 370:59-67.

28. Jay N, Berry JM, Hogeboom CJ, et al. Colposcopic appearance of anal squamous intraepithelial lesions: relationship to histopathology. Dis Colon Rectum 1997;40:919-28.

29. Lam JM, Hoch JS, Tinmouth J, et al. Cost-effectiveness of screening for anal precancers in HIV-positive men. AIDS 2011;25:635-42.

30. Palefsky JM, Rubin M. The epidemiology of anal human papillomavirus and related neoplasia. Obstet Gynecol Clin North Am 2009;36:187-200.

31. Salit IE, Lytwyn A, Raboud J, et al. The role of cytology (Pap tests) and human papillomavirus testing in anal cancer screening. AIDS 2010;24:1307-13.

32. Salit IE, Tinmouth J, Chong S, et al. Screening for HIV-associated anal cancer: correlation of HPV genotypes, p16, and E6 transcripts with anal pathology. Cancer Epidemiol Biomarkers Prev 2009; 18:1986-92.
33. Machalek DA, Poynten M, Jin F, et al. Anal human papillomavirus infection and associated neoplastic lesions in men who have sex with men: a systematic review and meta-analysis. Lancet Oncol 2012;13:487-500.

34. McCredie MR, Sharples KJ, Paul C, et al. Natural history of cervical neoplasia and risk of invasive cancer in women with cervical intraepithelial neoplasia 3: a retrospective cohort study. Lancet Oncol 2008;9:425-34

35. Scholefield JH, Castle MT, Watson NF. Malignant transformation of high-grade anal intraepithelial neoplasia. Br J Surg 2005; 92:1133-6.

36. Watson AJ, Smith BB, Whitehead MR, et al. Malignant progression of anal intra-epithelial neoplasia. ANZ J Surg 2006;76:715-7.

37. Botes LP, Pett S, Carr A, et al. Anal cytological abnormalities are poor predictors of high-grade intraepithelial neoplasia amongst HIV-positive men who have sex with men. Sex Health 2013;10:9-17.

38. Howard K. The cost-effectiveness of screening for anal cancer in men who have sex with men: a systematic review. Sex Health 2012;9:610-9.

39. Scholefield AM, Sukthankar A, Desai M, et al. OC-071 Screening for anal pre-cancer in HIV positive and negative men who have sex with men (MSM) and renal transplant recipients: early experience from a Manchester based prospective study. Gut 2014;63(Suppl):A35-6.

40. Nielson CM, Harris RB, Nyitray AG, et al. Consistent condom use is associated with lower prevalence of human papillomavirus infection in men. $J$ Infect Dis 2010;202:445-51.

41. Bleeker MC, Hogewoning CJ, Voorhorst FJ, et al. Condom use promotes regression of human papillomavirus-associated penile lesions in male sexual partners of women with cervical intraepithelial neoplasia. Int J Cancer 2003;107:804-10.

42. Holly EA, Whittemore AS, Aston DA, et al. Anal cancer incidence: genital warts, anal fissure or fistula, hemorrhoids, and smoking. J Natl Cancer Inst 1989;81:1726-31.

43. Etienney I, Vuong S, Daniel F, et al. Prevalence of anal cytologic abnormalities in a French referral population: a prospective study with special emphasis on HIV, HPV, and smoking. Dis Colon Rectum 2008;51:67-72.

44. Castellsague X, Bosch FX, Munoz N, et al. Male circumcision, penile human papillomavirus infection, and cervical cancer in female partners. New Engl J Med 2002;346:1105-12.

45. Larke NL, Thomas SL, dos Santos Silva I, et al. Male circumcision and penile cancer: a systematic review and meta-analysis. Cancer Causes Contr 2011;22:1097.110.

46. Chih HJ, Lee AH, Colville L, et al. A review of dietary prevention of human papillomavirus-related infection of the cervix and cervical intraepithelial neoplasia. Nutr Cancer 2013;65:317-28.

47. Garland SM, Hernandez-Avila M, Wheeler CM, et al. Quadrivalent vaccine against human papillomavirus to prevent anogenital diseases. N Engl J Med 2007;356:1928-43.

48. FUTURE II Study Group. Quadrivalent vaccine against human papillomavirus to prevent high-grade cervical lesions. $N$ Engl J Med 2007;356:1915-27.

49. Palefsky JM, Giuliano AR, Goldstone S, et al. HPV vaccine against anal HPV infection and anal intraepithelial neoplasia. N Engl J Med 2011;365:1576-85.

50. Sobhani I, Walker F, Roudot-Thoraval F, et al. Anal carcinoma: incidence and effect of cumulative infections. AIDS 2004; 18:1561-9.

51. Devaraj B, Cosman BC. Expectant management of anal squamous dysplasia in patients with HIV. Dis Colon Rectum 2006; 49:36-40.

Affiliations: Division of Infectious Diseases (Medford), University of Ottawa, Ottawa, Ont.; Division of Infectious Diseases (Salit), University Health Network, University of Toronto, Toronto, Ont.

Contributors: Richard Medford reviewed all of the relevant publications in the English literature, extracted that information and wrote the initial draft of the article. Irving Salit concieved the idea for the article and revised the article for important intellectual content. Both of the authors approved the final version submitted for publication. 